\title{
A comparison of three semi-selective media for the isolation of methicillin-resistant Staphylococcus aureus
}

\author{
JANET L. ALLEN, M. E. COWAN and P. M. COCKROFT \\ Infection Control Department, St Mary's General Hospital, Milton Road, Portsmouth, Hants PO3 $6 A Q$
}

\begin{abstract}
Summary. During the 1980s the emergence of multiple antibiotic-resistant strains of Staphylococcus aureus posed new problems for infection control worldwide, many of which still remain. In investigations of outbreaks of infection, the laboratory has a key role in identifying infected or colonised patients and staff. The rapid isolation and accurate identification of the causative organisms are essential for the implementation of appropriate control measures. Speed and accuracy in identification, by colonial morphology, is often difficult to achieve in the presence of a mixed population of commensal bacteria.

To this end, the sensitivity of three media for the isolation of methicillin-resistant $S$. aureus (MRSA) from simulated clinical specimens was compared. Initial colonial recognition of MRSA was enhanced on methicillin-milk agar when compared with that on other media.
\end{abstract}

\section{Introduction}

Various techniques have been developed over the last decade for the identification of methicillinresistant Staphylococcus aureus (MRSA). Generally they have been adapted from methods which are now widely established in biotechnology. Gene probes, ${ }^{1}$ polymerase chain reaction ${ }^{2}$ and monoclonal antibody techniques ${ }^{3}$ provide greater accuracy but at present remain beyond the economic resources of the average diagnostic laboratory. Traditional but slower culture methods are usually the only available option. These techniques are on occasion unreliable, because they depend on the subjective visual assessment of the colonial appearance of organisms. Thus, the choice of a primary isolation medium becomes critical. The Combined Working Party of the Hospital Infection Society and the British Society for Antimicrobial Chemotherapy in their "Guidelines for the control of epidemic methicillin-resistant Staphylococcus aureus" (EMRSA $)^{4}$ recommended the use of either methicillinblood agar or methicillin-nutrient agar for the isolation of this organism. Local experience highlighted difficulties in the early recognition of suspected MRSA colonies with either medium. A review of published reports suggested that colonial recognition might be simplified by changing to alternative media previously developed for the routine isolation of staphylococci. Of those available, milk agar ${ }^{5}$ had the advantage that it was easy and economical to produce. It was also noted that on a medium containing full fat milk, the majority of $S$. aureus strains produce colonies with enhanced pigmentation. ${ }^{6}$ This is a result of the interaction between the carotenoids present in the bacteria and the phospholipids and lipids in the milk. The pigmentation ranges in colour from cream to orange and is a useful identification marker for the organism in the presence of a heavy mixed flora.

The purpose of this study was to compare the ease of identification and isolation of MRSA on methicillinmilk agar with that on methicillin-blood agar and on Chapman's medium ${ }^{7}$ with added methicillin.

\section{Materials and methods}

\section{Isolation media}

Preliminary work suggested that the most reproducible results in terms of colony size, colour intensity and ease of colonial identification were found on a medium containing ultra-heat treated full-fat milk, which is readily available from any supermarket. Methicillin-milk agar was prepared from Taiyo Agar (Lucas Meyer) 2\% supplemented with milk $33 \% \mathrm{v} / \mathrm{v}$, $\mathrm{NaCl}$ (GPR; BDH) 4.5\% w/v, Nutrient Broth No. 2 (Unipath) $2.5 \% \mathrm{v} / \mathrm{v}$ and methicillin (Sigma) $4 \mathrm{mg} / \mathrm{L}$. Taiyo agar is made from Japanese seaweed and is used in this laboratory as a gelling agent. Chapman's medium was prepared from Taiyo agar $1.5 \%$ supplemented with Lab Lemco (Unipath) $0.1 \% \mathrm{w} / \mathrm{v}$, Bacteriological Peptone (Unipath) $1 \% \mathrm{w} / \mathrm{v}, \mathrm{NaCl} 7 \%$ w/v, Mannitol (Analar; BDH) $1 \% \mathrm{w} / \mathrm{v}$, phenol red solution (BDH) $0.0025 \% \mathrm{v} / \mathrm{v}$ and methicillin $4 \mathrm{mg} / \mathrm{L}$. Methicillin-blood agar was prepared from Columbia Agar (Unipath) $3.5 \%$ supplemented with defibrinated horse blood $8 \% \mathrm{v} / \mathrm{v}$ and methicillin $4 \mathrm{mg} / \mathrm{L}$. All plates were dried overnight at $37^{\circ} \mathrm{C}$ and stored at $4{ }^{\circ} \mathrm{C}$ until required. 
Table I. Comparison of the viable counts of MRSA on the three selective media after incubation for $48 \mathrm{~h}$ under optimal laboratory conditions

\begin{tabular}{lccccc}
\hline & \multicolumn{5}{c}{ Average viable count (cfu/ml) } \\
\cline { 2 - 6 } $\begin{array}{l}\text { Test } \\
\text { organisms }\end{array}$ & $\begin{array}{c}\text { Original } \\
\text { inoculum }\end{array}$ & $\begin{array}{c}\text { Blood } \\
\text { agar }\end{array}$ & $\begin{array}{c}\text { Methicillin- } \\
\text { blood agar }\end{array}$ & $\begin{array}{c}\text { Methicillin- } \\
\text { milk agar }\end{array}$ & $\begin{array}{c}\text { Chapman's medium } \\
\text { with methicillin }\end{array}$ \\
\hline MRSA & $10^{2}$ & $5.0 \times 10^{2}$ & $0.5 \times 10^{2}$ & $6.0 \times 10^{2}$ & $0.9 \times 10^{2}$ \\
(16 strains) & $10^{4}$ & $3.5 \times 10^{4}$ & $0.9 \times 10^{4}$ & $5.0 \times 10^{4}$ & $0.8 \times 10^{4}$ \\
& $10^{6}$ & $5.0 \times 10^{6}$ & $0.8 \times 10^{6}$ & $3.5 \times 10^{6}$ & $0.8 \times 10^{6}$ \\
EMRSA & $10^{2}$ & $4.0 \times 10^{2}$ & $0.7 \times 10^{2}$ & $2.0 \times 10^{2}$ & $0.5 \times 10^{2}$ \\
(14 strains) & $10^{4}$ & $3.0 \times 10^{4}$ & $0.7 \times 10^{4}$ & $5.0 \times 10^{4}$ & $0.8 \times 10^{4}$ \\
& $10^{6}$ & $2.5 \times 10^{6}$ & $0.8 \times 10^{6}$ & $1.0 \times 10^{6}$ & $0.8 \times 10^{6}$ \\
\hline
\end{tabular}

\section{Organisms}

Epidemic MRSA strains (EMRSA) and sporadic isolates (SMRSA) were supplied by the Staphylococcal Reference Laboratory, Central Public Health Laboratory, Colindale Avenue, London. Clinical isolates of Proteus spp., Escherichia coli, Klebsiella spp., Pseudomonas aeruginosa, Flavobacterium spp., aerobic spore-bearers and methicillin-resistant coagulasenegative staphylococci (MRCNS) were used to prepare the simulated clinical specimens.

\section{Recovery of pure cultures of $M R S A$}

Dilutions of each test MRSA strain were prepared in sterile Ringer's solution (quarter-strength) to give working concentrations of $c .10^{2}, 10^{4}$ and $10^{6} \mathrm{cfu} / \mathrm{ml}$. Plates were inoculated with an automatic pipette and were read after incubation under optimal conditions for 20 and $48 \mathrm{~h}$ : methicillin-blood agar at $30^{\circ} \mathrm{C}$, methicillin-milk agar and Chapman's medium at $35^{\circ} \mathrm{C}$, which are the optimal temperatures for the growth of $S$. aureus on these media. ${ }^{7.8}$

\section{Recovery of MRSA from simulated clinical specimens}

The specimens were prepared to resemble, as closely as possible, the mixed flora previously observed during outbreak investigations. Overnight broth cultures of each organism were diluted in sterile Ringer's solution (quarter strength) to give a working concentration of c. $10^{8} \mathrm{cfu} / \mathrm{ml}$. The simulated clinical samples were prepared individually by adding varying volumes of these dilutions to sterile quarter-strength Ringer's solution. Plates were inoculated directly from the sample tube with an automatic pipette to minimise sampling error and plated for single colonies. A panel of 10 laboratory staff read the plates after incubation for 20 and $48 \mathrm{~h}$. The panel were unaware of the contents of the specimens. All were shown pure isolates of MRSA on each of the test media prior to the reading. Results were compared by means of a formula described by Goldschmidt and co-workers. ${ }^{9}$

Sensitivity of the test $=$

Number of true positives

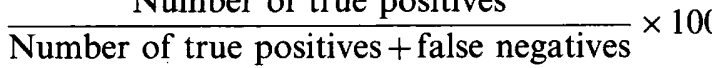

\section{Confirmatory tests for identification}

All suspected isolates of MRSA were subjected to confirmatory identification tests. A commercial coagglutination test (Staphylase; Unipath) was the initial screening test for isolates from both methicillin-milk and methicillin-blood agar. The "Rapidec" test (bioMérieux, France) was used to confirm identification if the co-agglutination test was equivocal. Methicillin resistance was confirmed by a standard disk sensitivity test by Stokes method ${ }^{\mathbf{1 0}}$ on isoSensitest agar (Unipath) supplemented with lysed horse blood $5 \%$. Plates were incubated overnight at $30^{\circ} \mathrm{C} .{ }^{8}$ The initial confirmatory identification test from Chapman's medium was the antibiotic sensitivity test followed by the co-agglutination test from the isoSensitest agar. ${ }^{11}$

\section{Results}

On methicillin-milk agar MRSA colonies vary in colour from cream to orange. The colour intensity deepens as incubation is prolonged. MRSA isolates on Chapman's medium produce yellow colonies provided that they use mannitol. On methicillin-blood agar, the pigmentation of MRSA colonies varies between strains and even within the same strain.

The results of the study on the recovery of pure MRSA isolates from the various media are shown in table $I$. The variation in results is not significant and is compatible with sampling error.

Seventy simulated clinical specimens were used in the main study, $29(41 \%)$ of which contained MRSA either in pure or mixed culture (table II). This is far in excess of the percentage isolation rate usually seen in outbreak investigations. The results of the study are shown in table II. A statistical comparison by the Kruskal-Wallis analysis of variance shows that methicillin-milk agar was more sensitive for the isolation of MRSA than methicillin-blood agar after incubation for 20 and $48 \mathrm{~h}(\mathrm{p}=<0.001$ and 0.005 0.001 respectively). Although there was no statistically significant difference between the sensitivities of methicillin-milk agar and Chapman's medium after incubation for 20 and $48 \mathrm{~h}(\mathrm{p}=0.975-0.05)$ (table II), 
Table II. Comparison of the isolation of MRSA from simulated clinical specimens expressed as the sensitivity of the test medium

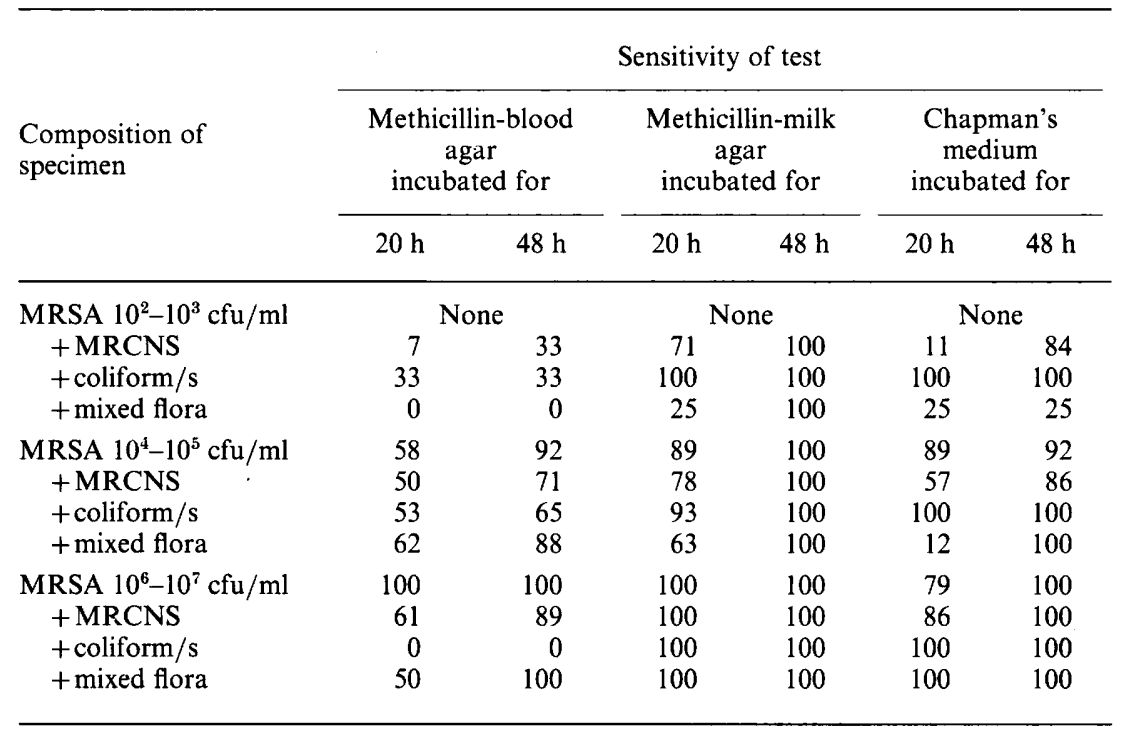

methicillin-milk agar was the only medium on which all the members of the panel were able to identify all the specimens containing MRSA (table II).

\section{Discussion}

The choice of initial identification tests relies on the user's ability to recognise a wide range of organisms by their colonial morphology. Specific tests on all isolates are rarely possible because of the cost and time involved. The choice of agar is, therefore, of fundamental importance, since it may facilitate presumptive identification at minimal cost in the shortest time. This applies particularly in large outbreaks when the onus is on the laboratory to identify any colonised or infected staff and patients as soon as possible to ensure that the appropriate infection control procedures are initiated rapidly.

Local experience in MRSA outbreaks suggested probable failures in isolating and identifying low numbers of MRSA in the presence of a heavy mixed commensal flora despite pre-incubating the specimens in salt broth $7 \% .{ }^{12}$ The aim of this study was to find a suitable isolation medium that would overcome these problems.

The advantage of methicillin-milk agar over Chapman's medium is that the colonial pigmentation does not rely on carbohydrate fermentation, which may be a variable factor. Colonial pigmentation on methicillin milk agar is more uniform in colour and easier to detect in the presence of a heavy mixed flora than on methicillin blood agar. Colonies of MRCNS on methicillin blood agar may closely resemble MRSA in colour; thus, the choice of colony for the coagglutination test may be difficult. If an MRCNS strain uses mannitol, the colonies will resemble MRSA on Chapman's medium. On methicillin-milk agar, MRCNS were, with one exception, white. Pigmented colonies of $S$. sciuri, an unusual skin commensal, superficially resembled those of MRSA on both methicillin-milk and methicillin-blood agar but all confirmatory identification tests for MRSA were negative.

The results from the viable count studies showed there was little to choose between the three media after incubation for $48 \mathrm{~h}$. After incubation for $20 \mathrm{~h}$, counting was difficult because of small colonial size; this was particularly noticeable on Chapman's medium. However, it is recommended that Chapman's medium is incubated for $36 \mathrm{~h}$ before the plates are examined. ${ }^{7}$ This has implications for its usefulness in outbreak investigations when the onus is on the laboratory to produce an early and accurate report. During this work, some difficulty was experienced with excessive variation in colonial morphology-this has also been reported by other workers. ${ }^{13}$ This problem was more noticeable with methicillin-blood agar and Chapman's medium.

Most of this work concentrated on the isolation and identification of MRSA, under laboratory controlled conditions, from a range of simulated clinical specimens. The sensitivity of each of the test media was determined after incubation for 20 and $48 \mathrm{~h}$. The only medium to achieve $100 \%$ sensitivity was methicillinmilk agar after incubation for $48 \mathrm{~h}$, although it has been suggested that overnight incubation is sufficient. ${ }^{4}$ In our experience, a primary incubation time of $20 \mathrm{~h}$ improves colonial pigmentation on both methicillinmilk and methicillin-blood agar. ${ }^{14}$ This facilitates the initial identification of MRSA, hence improving the sensitivity of the test. The failure to identify any colonised or infected person will prolong an outbreak with accompanying cost implications for the laboratory and hospital concerned. 
All colonies isolated on methicillin-milk agar, which were subjected to co-agglutination tests proved to be MRSA. Eighty-five percent of confirmatory tests on isolates from Chapman's medium were positive but only $65 \%$ from methicillin-blood agar. This confirms that the initial colonial recognition of MRSA on methicillin-milk agar is easier than on either of the other media. Therefore, methicillin-milk agar represents a saving in laboratory time and costs by reduced use of confirmatory tests.

In this laboratory, methicillin milk agar is now used as a primary isolation medium for MRSA. It still requires further validation by its application in a large outbreak, but results so far have been encouraging. During a small outbreak, methicillin-milk and methicillin-blood agar were used in parallel and, in two cases, colonisation with MRSA was detected only on methicillin-milk agar. This supports the original hypothesis that MRSA may not be recognised easily solely by colonial morphology on methicillin-blood

\section{References}

1. Archer GL, Pennell E. Detection of methicillin resistance in staphylococci by using a DNA probe. Antimicrob Agents Chemother 1990; 34: 1720-1724.

2. Murakami K, Minamide W, Wada K, Nakamura E, Teraoka H, Watanabe S. Identification of methicillinresistant strains of staphylococci by polymerase chain reaction. J Clin Microbiol 1991; 29 : 2240-2244.

3. Harrington CR, O'Hara DM, Reynolds PE. Characterisation of a monoclonal antibody and its use in the immunoaffinity purification of penicillin-binding protein $2^{\prime}$ of methicillinresistant Staphylococcus aureus. FEMS Microbiol Lett 1989; 65 : 143-147.

4. Report of a Combined Working Party of the Hospital Infection Society and British Society for Antimicrobial Chemotherapy. Revised guidelines for the control of epidemic methicillin-resistant Staphylococcus aureus. J Hosp Infect 1990; 16: 351-377.

5. Christie R, Keogh EV. Physiological and serological characteristics of staphylococci of human origin. J Pathol Bacteriol $1940 ; 51$ : 189-197.

6. Willis AT, O'Connor JJ, Smith JA. Colonial pigmentation of Staphylococcus aureus. J Pathol Bacteriol 1966; 92: 97-106.

7. Chapman GH. An improved Stone medium for the isolation and testing of food-poisoning staphylococci. Food Research 1948; 13: 100-105. agar when present in a heavy mixed commensal flora. Methicillin-milk agar is now accepted by the staff as a valuable medium for identification and isolation of MRSA.

Recently the tendency in microbiology has been towards the use of chemically defined media for the identification and isolation of an ever increasing range of bacteria which may cause infection. In this study, the use of methicillin-milk agar was a re-appraisal of an old established medium, although ultra-heat treated milk was used in place of sterilised milk. It returns to the concept of a "simple" medium which is relatively cheap to produce, and, in this laboratory, has proved to be a significant practical advance in the laboratory management of MRSA outbreaks.

We are grateful to Dr R. R. Marples of the Staphylococcal Reference Laboratory, CPHL Colindale Avenue, London, for kindly supplying the reference strains of MRSA, the PHL Portsmouth Laboratory staff who read the plates and Mr D. J. Lavis who patiently prepared the media.

8. Garrod LP, Lambert HP, O'Grady F. In: Antibiotics and chemotherapy, 5th edn. Edinburgh, Churchill Livingstone. $1981: 474$.

9. Goldschmidt MC, Fung DY, Grant R, White J, Brown T. New aniline blue dye medium for rapid identification and isolation of Candida albicans. J Clin Microbiol 1991; 29: 1095-1099.

10. Report of the Working Party on Antibiotic Sensitivity Testing of the British Society for Antimicrobial Chemotherapy. A guide to sensitivity testing. J Antimicrob Chemother 1991; 27 (Suppl D): 1-50.

11. Flournoy DJ, Wongpradit S, Silberg SL. Screening media for detection of methicillin-resistant Staphylococcus aureus from non-sterile body sites. Med Microbiol Immunol 1990; 179: $25-30$.

12. Cookson B, Peters B, Webster M, Phillips I, Rahman M, Noble W. Staff carriage of epidemic methicillin-resistant Staphylococcus aureus. J Clin Microbiol 1989; 27: 1471-1476.

13. Lally RT, Ederer MN, Woolfrey BF. Evaluation of mannitol salt agar with oxacillin as a screening medium for methicillin-resistant Staphylococcus aureus. J Clin Microbiol 1985; 22 : 501-504.

14. Cruickshank R, Duguid JP, Swain RHA. In: Medical microbiology, a guide to the laboratory diagnosis and control of infection, 11th edn. Edinburgh, Churchill Livingstone. $1965: 748$. 